\title{
Improvement of model evaluation by incorporating prediction and measurement uncertainty
}

\author{
Lei Chen, Shuang Li, Yucen Zhong, and Zhenyao Shen \\ State Key Laboratory of Water Environment Simulation, School of Environment, \\ Beijing Normal University, Beijing, 100875, PR China
}

Correspondence: Zhenyao Shen (zyshen@bnu.edu.cn)

Received: 6 February 2017 - Discussion started: 23 August 2017

Revised: 19 June 2018 - Accepted: 24 July 2018 - Published: 3 August 2018

\begin{abstract}
Numerous studies have been conducted to assess uncertainty in hydrological and non-point source pollution predictions, but few studies have considered both prediction and measurement uncertainty in the model evaluation process. In this study, the cumulative distribution function approach (CDFA) and the Monte Carlo approach (MCA) were developed as two new approaches for model evaluation within an uncertainty condition. For the CDFA, a new distance between the cumulative distribution functions of the predicted data and the measured data was established in the model evaluation process, whereas the MCA was proposed to address conditions with dispersed data points. These new approaches were then applied in combination with the Soil and Water Assessment Tool in the Three Gorges Region, China. Based on the results, these two new approaches provided more accurate goodness-of-fit indicators for model evaluation compared to traditional methods. The model performance worsened when the error range became larger, and the choice of probability density functions (PDFs) affected model performance, especially for non-point source (NPS) predictions. The case study showed that if the measured error is small and if the distribution can be specified, the CDFA and MCA could be extended to other model evaluations within an uncertainty framework and even be used to calibrate and validate hydrological and NPS pollution (H/NPS) models.
\end{abstract}

\section{Introduction}

Prediction of non-point source (NPS) pollution has become increasingly utilized because NPS pollution is a key threat to bodies of water (Shen et al., 2014). Numerous hydrological models, including the Soil and Water Assessment Tool (SWAT), the Hydrological Simulation ProgramFortran (HSPF) and the Agricultural Non-Point Source Model (AGNPS), have been developed and widely applied to hydrological and NPS (H/NPS) pollution analyses and watershed management (Yang et al., 2008). NPS pollution is reportedly driven by random and diffuse factors, such as climate, land use, soil, vegetation cover and human activities (Ouyang et al., 2009), and model confidence in NPS prediction, represented by model calibration and validation, is currently lacking in modelling research.

Hydrological models always require input data, optimal parameters and proper model structure (Di Baldassarre and Montanari, 2009), whereas data measurement often involves processes of sampling, transportation and analyses. Errors in these complex processes lead to uncertainty in the data (Chaney et al., 2015). Uncertainties in hydrology and NPS modelling are classified as either measurement uncertainty or prediction uncertainty (Chen et al., 2015; Di Baldassarre and Montanari, 2009). Uncertainty analysis is a crucial step in the application of hydrological models (Guinot et al., 2011). The uncertainty surrounding model structure and parameterization has been extensively investigated (Wu et al., 2017). Several approaches, including the generalized likelihood uncertainty estimation (GLUE) (Hassan et al., 2008; Sathyamoorthy et al., 2014; Cheng et al., 2014), the Bayesian approach (Freni and Mannina, 2010; Han and Zheng, 2016; Parkes and Demeritt, 2016; Zhang et al., 2009a), sequential uncertainty fitting (SUFI-2) (Vilaysane et al., 2015; Abbaspour et al., 2007), and Markov chain Monte Carlo (MCMC) (Vrugt et al., 2003; Zhang et al., 2016), have been proposed. However, 
due to the lack of data, relatively few studies have focused on the inherent uncertainty in measured data, and even fewer studies have considered measurement and prediction uncertainties in the evaluation of model performance (Di Baldassarre and Montanari, 2009; Montanari and Di Baldassarre, 2013).

In model evaluation, calibration is the process used to generate optimal parameters for the best goodness-of-fit between the predicted data and the measured data, and validation is the process of checking the model performance using another series of measured data (Chen et al., 2014). Traditional model evaluation only considers the goodness-of-fit between sets of measured data points and predicted data points (Westerberg et al., 2011). Such point-to-point methods might be inadequate because they fail to incorporate the existing uncertainties mentioned above. Previous studies have noted that if prediction uncertainty exists, the predicted data could be expressed as a confidence interval (CI) or a probability density function (PDF) (Franz and Hogue, 2011; Shen et al., 2012). Harmel and Smith (2007) used the probable error range (PER) as an expression of measurement uncertainty and modified the goodness-of-fit indicators using the deviation term between the predicted data points and the nearest measurement uncertainty boundaries. Harmel et al. (2010) further modified this deviation term using a correction factor, which was determined by the degree of overlap between each pair of measured and predicted intervals. This idea is instructive, but it might be questionable sometimes because a larger uncertainty or error would result in more overlap between the prediction and measurement intervals, which would indicate better model performance. In this regard, Chen et al. (2014) developed an interval-deviation approach (IDA), which demonstrated that H/WQ models should be evaluated against both the nearest and farthest boundaries (the inherent uncertainty intervals). Generally, this IDA approach is suitable for incomplete data conditions, but when more data could be collected or when a continuous and random data distribution could be assumed, these intervals may not always be practical. Current research tends to express uncertain data as certain function distributions to express an error term (Zhang et al., 2009b), which might lead to a more feasible expression than either the traditional or IDA methods.

The objective of this study is to develop a new framework for model evaluation by incorporating prediction and measurement uncertainty. Two methods, the cumulative distribution function approach (CDFA) and the Monte Carlo approach (MCA), were proposed for different situations (Sect. 2). Then, the new methods were used in combination with the SWAT to evaluate the Three Gorges Reservoir Area (TGRA), China, as a case study (Sects. 3 and 4).

\section{Methodology}

In this study, the Nash-Sutcliffe efficiency (NSE) coefficient was selected from commonly used indicators, and the expression is as follows:

$\mathrm{NSE}=1-\sum_{i=1}^{N}\left(O_{i}-P_{i}\right)^{2} / \sum_{i=1}^{N}\left(O_{i}-\bar{O}_{i}\right)^{2}$,

where $\left\{O_{i} \mid i=1,2, \ldots, N\right\}$ is the set of measured data, $\left\{P_{i} \mid i=1,2, \ldots, N\right\}$ is the set of predicted data and $\bar{O}$ is the mean value of the measured data.

In traditional indicators, the deviation between the measured and predicted data is expressed by the absolute distance $\left(O_{i}-P_{i}\right)$ between the paired data points. This method is questionable because it fails to incorporate prediction and measurement uncertainty. In this paper, the probability distributions of each data set were statistically estimated, and the calculations of $O_{i}-P_{i}$ were modified by using stochastic distances between the paired PDFs. For the CDFA, cumulative distribution functions were used to describe uncertain data because they are simple and do not depend on the distributional properties throughout the data sets (see Sect. 2.1). A topological distance, which is based on the distance between cumulative distribution functions (distribution-to-distribution), was proposed to replace the traditional error item in the model evaluation. The Monte Carlo method was also used to generate groups of discrete uncertain data throughout the sampling process (Vrugt and Ter Braak, 2011). Thus, the MCA was proposed as a supplement to the CDFA when the uncertain data were discrete or when no specific distributions could be used (see Sect. 2.2). A flowchart of the model evaluation within the uncertainty framework is presented in Fig. 1.

\subsection{The description of the CDFA method}

The idea behind the CDFA was to replace the point-to-point comparison with the deviation between uncertain measured data and predicted data expressed as cumulative distribution functions. In fact, this is a modification of traditional goodness-of-fit indicators by replacing the calculations of their $O_{i}-P_{i}$ term by using stochastic distances between the paired probability density functions (PDFs). A topological distance was proposed, and visualizations of the topological distance are illustrated in Fig. 2. The cumulative distribution function was chosen because it is a monotone increasing function with a limited threshold and an integral property. The distance between cumulative distributions was then transformed into an area topological distance $(D)$. The proof of the rationality behind the topological distance $D$ is shown in Table 1 (in the Supplement).

Based on Table $1,(B, D)$ is a reasonable metric space, and $D$ is a reasonable measurement of $B$. Therefore, the difference between $F_{p}(x)$ and $F_{o}(x)$ is reasonable and advisable.

The detailed steps of the CDFA are as follows: 


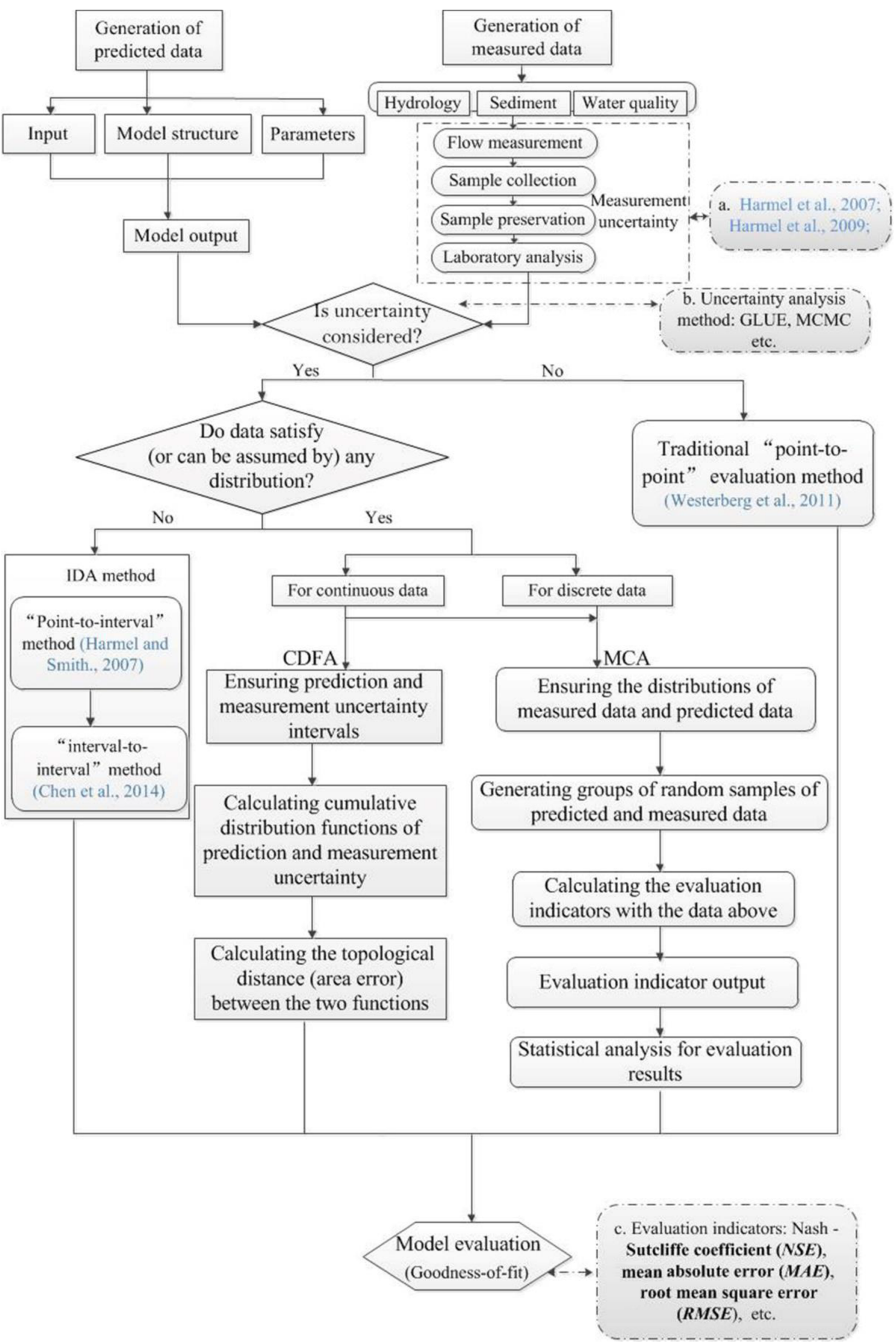

Figure 1. A general flowchart of model evaluation within the uncertainty framework. 
Table 1. The proof for the rationality of topological distance $D$.

\begin{tabular}{|c|c|c|}
\hline Objective & Implementation & Constraints \\
\hline The definition of $D$ & Defined $\forall f \in A$ & The changing of it $(f)$ is denoted by $g$. \\
\hline \multirow[t]{4}{*}{ The definition of $g$} & $f^{\prime}(x)= \begin{cases}0 & \left(x<x_{1}\right) \\
f(x) & \left(x_{1} \leq x \leq x_{2}\right) \\
1 & \left(x>x_{2}\right)\end{cases}$ & $\exists x_{1} x_{2} \in R f(x)=0$ and $f(x)=1$, then $\left(x_{1}<x_{2}\right)$ \\
\hline & $f^{\prime}(x)= \begin{cases}0 & \left(x<x_{1}\right) \\
f(x) & \left(x \geq x_{1}\right)\end{cases}$ & $\exists x_{1} \in R f(x)=0$ and $\exists ! x_{2} \in R, f(x)=1$ \\
\hline & $f^{\prime}(x)= \begin{cases}f(x) & \left(x \leq x_{2}\right) \\
1 & \left(x>x_{2}\right)\end{cases}$ & $\exists x_{2} \in R, f(x)=1$ and $\exists ! x_{1} \in R, f(x)=0$; \\
\hline & $f^{\prime}(x)=f(x)$ & $\exists ! x_{1} x_{2} \in R, f(x)=0$ and $f(x)=0$ \\
\hline The definition of $B$ & $B=\{h \mid h=g(f(x))\}, B \neq \varnothing$ & $h$ is a continuous function. \\
\hline \multirow[t]{2}{*}{ The definition of topological distance $D$} & \multirow{2}{*}{$\begin{array}{l}D=\int_{a}^{b}\left|f_{1}-f_{2}\right| \\
\forall D \notin\left\{D\left|D=\int_{a}^{b}\right| f_{1}-f_{2} \mid d_{x}, \forall f_{1}, f_{2} \in B\right\}\end{array}$} & $a<b, a, b \in R, a$ and $b$ are all real numbers*; \\
\hline & & $\exists m \in R ; m>D$ ( $D$ is a limited value $)$ \\
\hline Proof of positive definiteness & $D(j-j)=0$ & $\forall j \in B,|j-j|=0$, then $D=\int_{a}^{b}|j-j| d_{x}=0$ \\
\hline Proof of symmetry & $D\left(j_{1}, j_{2}\right)=D\left(j_{2}, j_{1}\right)$ & $\forall j_{1}, j_{2} \in B$ then, $\left|j_{1}-j_{2}\right|=\left|j_{2}-j_{1}\right|$ \\
\hline Trigonometric inequality & $\begin{array}{l}\left|j_{1}-j_{3}\right|=\left|j_{1}-j_{2}+j_{2}-j_{3}\right| \leq\left|j_{1}-j_{2}\right|+\left|j_{2}-j_{3}\right| \\
D\left(j_{1}, j_{3}\right) \leq D\left(j_{1}, j_{2}\right)+D\left(j_{2}, j_{3}\right)\end{array}$ & $\begin{array}{l}\forall j_{1} j_{2} j_{3} \in B ; \\
\int_{a}^{b}\left|j_{1}-j_{3}\right| d_{x} \leq \int_{a}^{b}\left(\left|j_{1}-j_{2}\right|+\left|j_{2}-j_{3}\right|\right) d_{x} \\
=\int_{a}^{b}\left|j_{1}-j_{2}\right| d_{x}+\int_{a}^{b}\left|j_{2}-j_{3}\right| d_{x}\end{array}$ \\
\hline
\end{tabular}

* Where the values of a and $\mathrm{b}$ are as far from the origin as possible; thus, the functions are integrated over a limited interval, and there are only small differences between the results and the results integrated for the real numbers $R$.
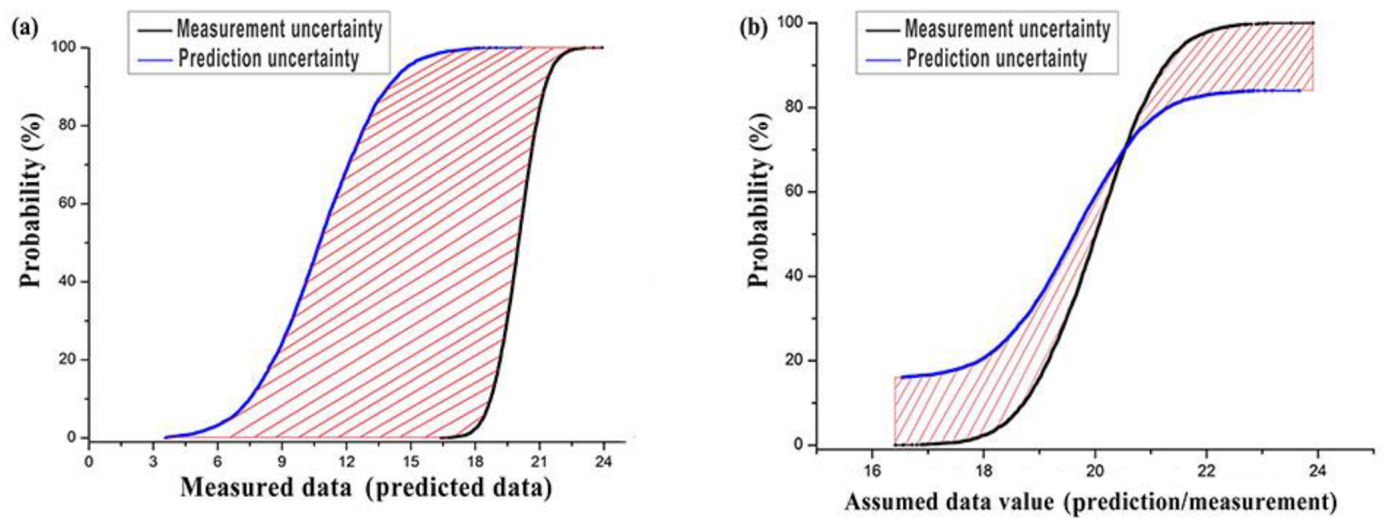

Figure 2. Expression of topological distance for (a) the case in which the measured and predicted data are non-overlapping and (b) the case in which the measured and predicted data are overlapping.

1. the prediction and measurement uncertainty are generated using GLUE, PER or other methods;

2. the prediction and measurement data intervals are analysed, and the cumulative distribution functions of the prediction uncertainty $\left(F_{o}(x)\right)$ and the measurement uncertainty $\left(F_{p}(x)\right)$ are calculated;

3. the topological interval (area distance) between the two functions $F_{o}(x)$ and $F_{p}(x)$ is quantified; and
4. the new $O_{i}-P_{i}$ is quantified, and the modified evaluation indicators are used for model evaluation.

\subsection{The MCA method}

In other cases, the measurement and prediction uncertainties might be expressed as discrete data, or no continuous distribution function may fit the data set. For example, even in some ideal conditions, the well-distributed gauges are available, the rain-gauge network cannot fully capture every point 
over the watershed and it is more common to have only a few stations distributed in space. Rainfall at unknown points is thus estimated by means of interpolation techniques. Some results showed that spatial interpolation techniques resulted in considerable uncertainty of rainfall spatial variability and transferred larger uncertainty to H/NPS modelling. In addition, Shen et al. (2013)'s study has been carried out into the effect of GIS data on water quality modelling and the uncertainty related to the combination of the available GIS maps. All these kinds of prediction uncertainty relating to limited model structures, or model input data, could result in discrete variables. To incorporate this type of uncertainty, MCA was implemented using the Monte Carlo technique, which has been used in many hydrological uncertainty studies (Sun et al., 2008; Zhang et al., 2016). The Monte Carlo technique is a type of random sampling method that considers combinations of different input components and determines a statistical distribution for the output data (Shen et al., 2013). A key step is sampling variables randomly for discrete data so that the measurement and prediction data can be expressed as certain distributions. Here, $\left(O_{i}-P_{i}\right)$ was replaced by a stochastic expression of the deviation between pairs of data groups, and these stochastic deviations were then used to calculate the evaluation indicators. The details of the MCA are as follows:

1. the distribution functions or discrete measured data points $\left(f_{o}(x)\right)$ and predicted data $\left(f_{p}(x)\right)$ are generated;

2. the sampling process of $\left.f_{p}(x)\right)$ and $f_{o}(x)$ is realized using the Latin hypercube sampling approach (Shen et al., 2012), and the Crystal Globe software was used to sample for the MCA;

3. based on the random samples of the predicted and measured data, corresponding individual goodness-of-fit indicators are calculated;

4. the sampling process is repeated until the target sample size is achieved; and

5. a group of goodness-of-fit indicator values are obtained, and these values are used to produce the statistical analysis for the model evaluation within the uncertainty framework.

\section{Case study}

In this study, the Daning Watershed, which is located in the central part of the TGRA, was selected as the study area. Previously, the uncertainty ranges related to the flow, sediment and TP predictions were quantified using the GLUE method (Chen et al., 2014; Shen et al., 2012), and these results and uncertainty ranges were used as the predicted data sets. Normal, uniform and lognormal distributions, which are classic and simple PDFs, were assumed for each predicted data set. More details about the uncertainty ranges and PDFs of the predicted flow, sediment and total phosphorus (TP) can be found in our previous study (Chen et al., 2014).

The measured streamflow, sediment and TP data at the Wuxi hydrological gauges were obtained from the Changjiang Water Resources Commission. Due to data limitations, the error range of the measured data was derived from Harmel et al. (2006, 2010) and Harmel and Smith (2007). Based on our previous study (Chen et al., 2014), the measurement uncertainty was assumed to be a normal distribution in this paper, and three scenarios, an ideal case, a typical case and a worst case, were used. The probable error ranges (PERs) for flow, sediment and TP were $2 \%$, $2 \%$ and $2 \%$, respectively, for each ideal-case scenario; $9 \%$, $16 \%$ and $26 \%$, respectively, for each typical-case scenario; and $36 \%, 102 \%$ and $221 \%$, respectively, for each worst-case scenario.

\section{Results and discussion}

\subsection{The model evaluation results using the CDFA}

The model evaluation results for flow, sediment and TP are shown in Table 2. For simplicity, only the NSE indicator was chosen as a model evaluation indicator, and the model evaluation results using a traditional point-to-point method were used as a baseline scenario. For the traditional method, the NSE values were $0.736,0.642$ and 0.783 for flow, sediment and TP, respectively. Using the CDFA method (assuming the measured error was small - the ideal case), the following changes to the NSE values were obtained: $0.752,0.660$ and 0.810 for flow, sediment and TP, respectively, in the normal distribution scenario; 0.742, 0.661 and 0.814 , respectively, in the uniform distribution scenario; and 0.752, 0.660 and 0.812 , respectively, in the lognormal distribution scenario. However, when the measurement error became large (for the typical case), the following NSE values were obtained: $0.751,0.657$ and 0.789 for flow, sediment and TP, respectively, in the normal distribution scenario; $0.742,0.661$ and 0.814 , respectively, in the uniform distribution scenario; and $0.751,0.657$ and 0.791 , respectively, in the lognormal distribution scenario. When the measurement error became negative (the worst-case scenario), the following NSE values were obtained: $0.744,0.551$ and -0.056 for flow, sediment and TP, respectively, in the normal distribution scenario; $0.736,0.545$ and -0.019 , respectively, in the uniform distribution scenario; and $0.744,0.437$ and -0.072 , respectively, in the lognormal distribution scenario.

\subsection{The model evaluation results using MCA}

The sampling size is important for MCA, so a sensitivity analysis was first conducted. Groups of $O_{i}$ and $P_{i}$ values (10, $50,100,200,500,1000,2000$ and 5000) were randomly generated and used to calculate the NSE, and the sampling sizes were obtained using statistical analysis of the NSE (only the 


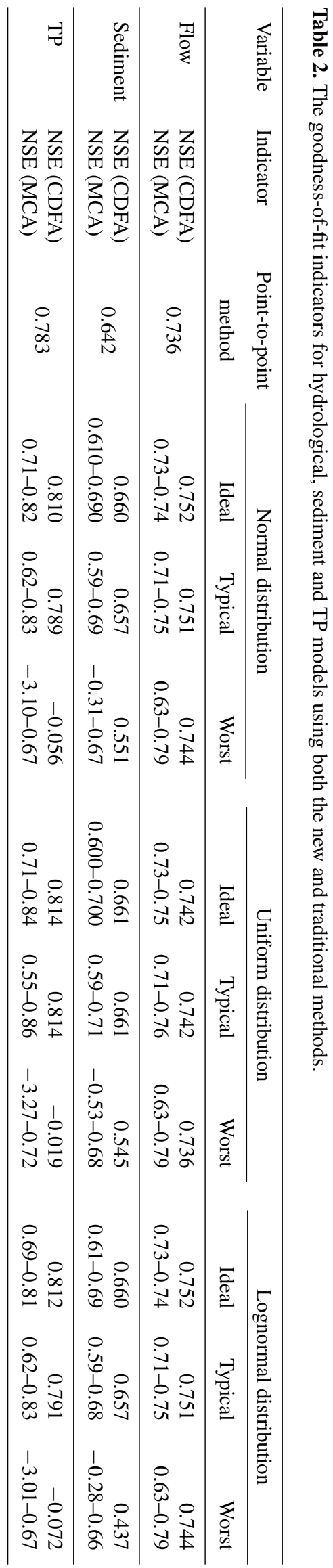

results for 1000, 2000, and 5000 are shown in Table 3). The sampling results showed that with increasing sampling size, the mean value and the coefficients of variation $\left(C_{\mathrm{V}}\right)$ of the flow, sediment and TP also increased. However, when the sampling sizes are larger than 2000 , the model performance becomes stable, and all indicators only changed within $1 \%$, indicating that larger sampling sizes of $O_{i}$ and $P_{i}$ would not further benefit the performance of the model. Thus, a sampling size of 5000 was chosen in this study.

The evaluation results, which are expressed as the $95 \%$ confidence interval of the NSE for the flow, sediment and TP predictions, are shown in Table 2. The NSE ranges for flow, sediment and TP in the ideal case were as follows: $0.73-0.74$, $0.61-0.69$ and $0.71-0.82$, respectively (normal distribution); $0.73-0.75,0.60-0.70$ and $0.71-0.84$, respectively (uniform distribution); and $0.73-0.74,0.61-0.69$ and $0.69-0.81$, respectively (lognormal distribution). The NSE ranges for flow, sediment and TP in the typical case were as follows: 0.71$0.75,0.59-0.69$ and $0.62-0.83$, respectively (normal distribution); $0.71-0.76,0.59-0.71$ and $0.55-0.86$, respectively (uniform distribution); and $0.71-0.75,0.59-0.68$ and $0.62-0.83$, respectively (lognormal distribution). The NSE ranges for flow, sediment and TP in the worst case were as follows: $0.63-0.79,-0.31-0.67$ and $-3.10-0.67$, respectively (normal distribution); $0.63-0.79,-0.53-0.68$ and $-3.27-0.72$, respectively (uniform distribution); and $0.63-0.79,-0.28$ 0.66 and $-3.01-0.67$, respectively (lognormal distribution).

\subsection{Analysis of influencing factors}

\subsubsection{Impact of error range}

Generally, the data uncertainty range should always be obtained by analysing a large amount of data, so it is difficult to ensure the error range of the predicted or measured data due to data limitations. In this study, the measurement error is expressed as the PER, and three PERs were obtained as expressions of different error ranges (Harmel and Smith, 2007). In this section, the error ranges of the measured data were assumed as the PERs, and the impacts of the PERs on the evaluation results of the CDFA and the MCA were quantified. Only the normal distribution was considered for the prediction data. For the ideal-case scenario (PER of $2 \%$ ), the NSE for the flow evaluation was 0.752 , but for the typicalcase and worst-case scenarios, the values of the NSE changed to 0.751 and 0.744 , respectively. Compared to the point-topoint result, the goodness-of-fit indicators obtained from the CDFA (NSE) increased by $21.3 \%$ for flow in the ideal case. The NSE increased by $20.1 \%$ and $9.8 \%$ for the typical-case and worst-case scenarios, respectively. Similar variations in the evaluation results were observed for the MCA method. The NSE for the flow evaluation was $63.5 \%$ for the ideal case (normal distribution) and was $40.9 \%$ and $10.6 \%$ for the typical case and the worst case, respectively (Harmel and Smith, 2007; Shen et al., 2013). For flow prediction, the evaluation 
Table 3. The result of sampling (2000 times and 5000 times) the flow, sediment and TP in different distributions.

\begin{tabular}{|c|c|c|c|c|c|c|c|c|}
\hline & & \multirow{2}{*}{$\begin{array}{l}\text { Number of } \\
\text { simulations }\end{array}$} & \multicolumn{2}{|c|}{ Normal distribution } & \multicolumn{2}{|c|}{ Uniform distribution } & \multicolumn{2}{|c|}{ Lognormal distribution } \\
\hline & & & $M_{\mathrm{V}}^{1}$ & $C_{\mathrm{V}}^{2}$ & $M_{\mathrm{V}}$ & $C_{\mathrm{V}}$ & $M_{\mathrm{V}}$ & $C_{\mathrm{V}}$ \\
\hline \multirow{9}{*}{ Flow } & \multirow{3}{*}{ Ideal } & 1000 & 0.738 & 0.005 & 0.742 & 0.006 & 0.736 & 0.005 \\
\hline & & 2000 & 0.737 & 0.005 & 0.742 & 0.006 & 0.736 & 0.005 \\
\hline & & 5000 & 0.737 & 0.005 & 0.742 & 0.006 & 0.736 & 0.005 \\
\hline & \multirow{3}{*}{ Typical } & 1000 & 0.734 & 0.013 & 0.736 & 0.013 & 0.735 & 0.014 \\
\hline & & 2000 & 0.734 & 0.013 & 0.738 & 0.013 & 0.732 & 0.013 \\
\hline & & 5000 & 0.733 & 0.013 & 0.737 & 0.013 & 0.733 & 0.013 \\
\hline & \multirow{3}{*}{ Worst } & 1000 & 0.578 & 0.055 & 0.824 & 0.014 & 0.713 & 0.015 \\
\hline & & 2000 & 0.683 & 0.047 & 0.748 & 0.013 & 0.730 & 0.013 \\
\hline & & 5000 & 0.693 & 0.048 & 0.737 & 0.013 & 0.737 & 0.013 \\
\hline \multirow{9}{*}{ Sediment } & \multirow{3}{*}{ Ideal } & 1000 & 0.643 & 0.029 & 0.657 & 0.043 & 0.641 & 0.028 \\
\hline & & 2000 & 0.642 & 0.030 & 0.657 & 0.044 & 0.642 & 0.028 \\
\hline & & 5000 & 0.642 & 0.030 & 0.657 & 0.044 & 0.642 & 0.029 \\
\hline & \multirow{3}{*}{ Typical } & 1000 & 0.639 & 0.039 & 0.652 & 0.05 & 0.64 & 0.057 \\
\hline & & 2000 & 0.640 & 0.038 & 0.654 & 0.049 & 0.637 & 0.038 \\
\hline & & 5000 & 0.638 & 0.039 & 0.653 & 0.049 & 0.638 & 0.038 \\
\hline & \multirow{3}{*}{ Worst } & 1000 & 0.378 & 0.818 & 0.484 & 0.987 & 0.467 & 1.224 \\
\hline & & 2000 & 0.440 & 0.713 & 0.440 & 0.906 & 0.415 & 1.21 \\
\hline & & 5000 & 0.446 & 0.717 & 0.433 & 0.914 & 0.424 & 1.213 \\
\hline \multirow{9}{*}{$\mathrm{TP}$} & \multirow{4}{*}{ Ideal } & 1000 & 0.773 & 0.038 & 0.782 & 0.044 & 0.771 & 0.039 \\
\hline & & 2000 & 0.772 & 0.039 & 0.782 & 0.044 & 0.771 & 0.039 \\
\hline & & 5000 & 0.771 & 0.039 & 0.781 & 0.045 & 0.772 & 0.040 \\
\hline & & 1000 & 0.744 & 0.074 & 0.747 & 0.106 & 0.747 & 0.106 \\
\hline & \multirow[t]{2}{*}{ Typical } & 2000 & 0.745 & 0.073 & 0.749 & 0.104 & 0.744 & 0.072 \\
\hline & & 5000 & 0.743 & 0.073 & 0.748 & 0.105 & 0.746 & 0.072 \\
\hline & \multirow{3}{*}{ Worst } & 1000 & -0.091 & -12.224 & -0.168 & -8.907 & -0.172 & -8.529 \\
\hline & & 2000 & -0.106 & -10.543 & -0.153 & -8.172 & -0.148 & -8.737 \\
\hline & & 5000 & -0.108 & -10.716 & -0.150 & -8.249 & -0.156 & -8.449 \\
\hline
\end{tabular}

${ }^{1} M_{\mathrm{V}}$ is the mean value ${ }^{2} C_{\mathrm{V}}$ is the coefficient of variation.

results obtained using the CDFA were all satisfactory with measurement error of any size; however, for the sediment and TP evaluations, the goodness-of-fit indicators became unacceptable if the measurement errors were large (in the worstcase scenario). In this regard, the range of measurement error showed different impacts on the flow, sediment and TP predictions. For example, the NSE values were $0.752,0.660$ and 0.810 for the flow, sediment and TP evaluations in the idealcase scenario. From the results above, a large measurement error would cause decreasing evaluation performance, which is different from the results of Harmel and Smith (2007). Similar results were observed for the MCA.

As shown in Table 2, increasing measurement error would lead to decreased NSE, which means less confidence in the model performance. The worst evaluation indicators were observed when the measurement error was the largest. The performance of the TP predictions became unacceptable when the PER was $221 \%$ (worst case). This result indicated that a threshold error range might exist for model evaluation. When the error range is less than this specific value (such as the ideal and typical cases for TP prediction used in this study), the model evaluation result is acceptable. However, if the measurement error exceeds this threshold value (worst case) the model evaluation would be unacceptable, and the confidence in the model performance would be lost, especially for the NPS prediction. However, in reality, it is often difficult to accurately measure pollutant data, especially in developing countries, such as China. In these countries, a "calibrated model" would have few advantages over an uncalibrated model because of the lack of precisely measured data.

\subsubsection{Impacts of data distribution}

The assignment of PDFs might be the most difficult and subjective task in the application of uncertainty analysis to hydrological models (Shen et al., 2013). Thus, model performance might be influenced not only by the error range, but also by the choice of PDF. In this study, the prediction uncertainty was assumed as three certain distribution functions, but it is always difficult to ensure which PDFs should be 
used (Shen et al., 2013). In this section, we further quantified the impacts of the different PDFs on model performance. For simplicity, only the results of the CDFA and typical-case scenario are considered here. For the flow evaluation, the NSE values were $0.751,0.742$ and 0.751 when the predicted data were modelled using the normal distribution, uniform distribution and lognormal distribution, respectively. For TP evaluation, the NSE values were $0.789,0.814$ and 0.791 for the normal distribution, uniform distribution and lognormal distribution, respectively. As in a previous study, the CI of the prediction was larger for flow than for TP (Shen et al., 2012). Thus, the prediction distributions have a low impact on the evaluation in cases of high CI values, but have a bigger impact on the evaluation when the CI of the prediction is low. Compared to the baseline scenario, the NSE values for the hydrological prediction increased by $20.1 \%, 7.3 \%$ and $20.3 \%$ for the normal, uniform and lognormal distributions, respectively. These results indicated that the choice of PDF would show certain impacts on the model evaluation for hydrological, sediment and TP applications. This result is consistent with previous studies, which also showed that prediction uncertainty distributions can affect the goodnessof-fit indicators (Harmel et al., 2010). Table 2 also indicates that the choice of predicted PDFs should be dependent on the selection of the measured PDFs. If the measurement and prediction uncertainties are set using the same PDFs, such as a normal distribution, the goodness-of-fit indicators would be larger, indicating a more reliable model performance. Thus, the choice of proper PDFs is important to make accurate model evaluation for NPS predictions. Based on these results, we suggest that model acceptability can be attained by using certain PDFs on the model output by collecting information from model documentation, previous studies, and other literature to make an "educated guess".

\subsection{Comparison with previous methods}

In a previous study, Harmel and Smith (2007) advanced the IDA method, and this "point-to-interval" method was based on the distance between the nearest boundaries of paired intervals. Compared to our results, the difference between the paired data intervals or the paired PDFs overlapping for larger uncertainties would be mistakenly regarded as "no difference". The point-to-interval method gave a higher goodness-of-fit, but the measured data were only treated as data points. In this regard, deviations between measurements and the prediction data would be ignored in the model evaluation, which is not appropriate because the measurement error range would greatly affect the model performance (as mentioned in Sect. 4.3).

Chen et al. (2014) improved the nearest method by correcting for the overlapping parts of the uncertainty data and using both the nearest and farthest boundaries. Using the IDA, the NSE for the hydrological prediction would be 0.834 in the ideal case. However, the CDFA method produced lower
NSE values, which were 0.752 for the normal distribution, 0.742 for the uniform distribution and 0.752 for the lognormal distribution. In the typical case, the IDA method would produce an NSE value of 0.833 , but the CDFA would result in an NSE value of 0.751 for the normal distribution, 0.742 for the uniform distribution, and 0.751 for the lognormal distribution. The difference between the IDA and the CDFA would be largest for the worst case, in which the NSE values would be 0.780 for the IDA method and 0.744 (normal distribution), 0.736 (uniform distribution), or 0.744 (lognormal distribution) for the CDFA method (all results of the IDA can be found in Chen et al., 2014).

In Chen et al. (2014), an "interval-to-interval" method was proposed in which an absolute distance between measurement and prediction uncertainty data was derived from both the nearest and farthest boundaries. However, due to data limitations, a weight factor was used to balance the nearest boundaries and the farthest boundaries, and the choice of weight factor was subjective. When the weight factor was set to 0.5 , the IDA method would produce similar goodnessof-fit indicators to the results of the CDFA using the uniform or normal distributions for both the predicted and measured data (Chen et al., 2014). For example, the NSE value for the hydrological prediction was 0.764 using the IDA method; if the CDFA was used, the goodness-of-fit indicators would be $0.752,0.742$, and 0.752 for the normal, lognormal, and uniform distributions, respectively. Therefore, when specific PDFs were used, the IDA method could be viewed as a simplification of the CDFA. Previous studies have also indicated that the lognormal distribution provides a relatively close approximation to the true error characteristics, so the CDFA could be more practical if certain prediction uncertainties exist (Shen et al., 2015).

\section{Conclusion}

In this study, two new methods were proposed and employed to evaluate model performance within an uncertainty framework: the CDFA and the MCA. Using the CDFA and the MCA, both prediction and measurement uncertainty could be considered for model evaluation in a post-calibration process, and the possible impacts of error range and the choice of PDFs could be quantified for a real application. Based on the results, the model performance worsened when a larger error range existed, and the choice of PDF affected the model performance, especially for NPS pollution predictions. These proposed methods could be extended to other goodness-of-fit indictors and other watershed models to provide a substitution for traditional model evaluations within an uncertainty framework. Thus, the new approaches could be a substitute for traditional goodness-of-fit indicators and they could be used for the model evaluation process. 
However, it should be noted that the proposed CDFA and the MCA would serve for model evaluation in a postcalibration process rather than a new calibration technique due to the technical complexity in implementing this approach within the model calibration. With the results presented, fixed PDFs or error range for prediction data could not be found due to insufficient knowledge and natural randomness. Thus modellers should better assess the error range of measured data for their use in watershed simulations, and more data should be gathered to obtain a real measurement error range and a proper PDF for the predicted data. Further explanations are also suggested for the inherent uncertainty of hydrological and pollutant transportation processes. More case studies should be conducted to test the IDA, CDFA and MCA in future practical analyses of other watershed models.

Data availability. The data could be obtained by emailing the correspondence author or the first author.

Supplement. The supplement related to this article is available online at: https://doi.org/10.5194/hess-22-4145-2018-supplement.

Author contributions. ZS gave the innovative idea of the new method, while LC and YZ originated the main framework of this algorithm. LC and SL wrote the main manuscript text and prepared figures. All the authors reviewed the manuscript and all take responsibility for it.

Competing interests. The authors declare that they have no conflict of interest.

Special issue statement. This article is part of the special issue "Coupled terrestrial-aquatic approaches to watershed-scale water resource sustainability". It is not associated with a conference.

Acknowledgements. This research was funded by the National Natural Science Foundation of China (nos. 51579011 and 51779010), the Newton Fund (grant ref: BB/N013484/1), the Key Laboratory of Nonpoint Source Pollution Control, Ministry of Agriculture, P. R. China (1610132016005), and the Interdiscipline Research Funds of Beijing Normal University.

Edited by: Alberto Guadagnini

Reviewed by: two anonymous referees

\section{References}

Abbaspour, K. C., Yang, J., Maximov, I., Siber, R., Bogner, K., Mieleitner, J., Zobrist, J., and Srinivasan, R.: Modelling hydrology and water quality in the pre-alpine/alpine Thur watershed using SWAT, J. Hydrol., 333, 413-430, 2007.

Chaney, N. W., Herman, J. D., Reed, P. M., and Wood, E. F.: Flood and drought hydrologic monitoring: the role of model parameter uncertainty, Hydrol. Earth Syst. Sci., 19, 3239-3251, https://doi.org/10.5194/hess-19-3239-2015, 2015.

Chen, L., Shen, Z., Yang, X., Liao, Q., and Yu, S. L.: An IntervalDeviation Approach for hydrology and water quality model evaluation within an uncertainty framework, J. Hydrol., 509, $207-$ $214,2014$.

Chen, L., Gong, Y., and Shen, Z.: A comprehensive evaluation of input data-induced uncertainty in nonpoint source pollution modeling, Hydrol. Earth Syst. Sci. Discuss., 12, 11421-11447, https://doi.org/10.5194/hessd-12-11421-2015, 2015.

Cheng, Q., Chen, X., Xu, C., Reinhardt-Imjela, C., and Schulte, A.: Improvement and comparison of likelihood functions for model calibration and parameter uncertainty analysis within a Markov chain Monte Carlo scheme, J. Hydrol., 519, 2202-2214, 2014.

Di Baldassarre, G. and Montanari, A.: Uncertainty in river discharge observations: a quantitative analysis, Hydrol. Earth Syst. Sci., 13, 913-921, https://doi.org/10.5194/hess-13-913-2009, 2009.

Franz, K. J. and Hogue, T. S.: Evaluating uncertainty estimates in hydrologic models: borrowing measures from the forecast verification community, Hydrol. Earth Syst. Sci., 15, 3367-3382, https://doi.org/10.5194/hess-15-3367-2011, 2011.

Freni, G. and Mannina, G.: Bayesian approach for uncertainty quantification in water quality modelling: The influence of prior distribution, J. Hydrol., 392, 31-39, 2010.

Guinot, V., Cappelaere, B., Delenne, C., and Ruelland, D.: Towards improved criteria for hydrological model calibration: theoretical analysis of distance- and weak form-based functions, J. Hydrol., 401, 1-13, 2011.

Han, F. and Zheng, Y.: Multiple-response Bayesian calibration of watershed water quality models with significant input and model structure errors, Adv. Water Resour., 88, 109-123, 2016.

Harmel, R. D. and Smith, P. K.: Consideration of measurement uncertainty in the evaluation of goodness-of-fit in hydrologic and water quality modeling, J. Hydrol., 337, 326-336, 2007.

Harmel, R. D., Cooper, R. J., Slade, R. M., Haney, R. L., and Arnold, J. G.: Cumulative uncertainty in measured streamflow and water quality data for small watersheds, T. ASABE, 49, 689701, 2006.

Harmel, R. D., Smith, P. K., and Migliaccio, K. W.: Modifying Goodness-of-Fit Indicators to Incorporate Both Measurement and Model Uncertainty in Model Calibration and Validation, T. ASABE, 53, 55-63, 2010.

Hassan, A. E., Bekhit, H. M., and Chapman, J. B.: Uncertainty assessment of a stochastic groundwater flow model using GLUE analysis, J. Hydrol., 362, 89-109, 2008.

Montanari, A. and Di Baldassarre, G.: Data errors and hydrological modelling: The role of model structure to propagate observation uncertainty, Adv. Water Resour., 51, 498-504, 2013.

Ouyang, W., Wang, X., Hao, F., and Srinivasan, R.: Temporalspatial dynamics of vegetation variation on non-point source nutrient pollution, Ecol. Model., 220, 2702-2713, 2009. 
Parkes, B. and Demeritt, D.: Defining the hundred year flood: A Bayesian approach for using historic data to reduce uncertainty in flood frequency estimates, J. Hydrol., 540, 1189-1208, 2016.

Sathyamoorthy, S., Vogel, R. M., Chapra, S. C., and Ramsburg, C. A.: Uncertainty and sensitivity analyses using GLUE when modeling inhibition and pharmaceutical cometabolism during nitrification, Environ. Model. Softw., 60, 219-227, 2014.

Shen, Z., Huang, Q., Liao, Q., Chen, L., Liu, R., and Xie, H.: Uncertainty in flow and water quality measurement data: A case study in the Daning River watershed in the Three Gorges Reservoir region, China, Desalin. Water Treat., 51, 3995-4001, 2013.

Shen, Z., Qiu, J., Hong, Q., and Chen, L.: Simulation of spatial and temporal distributions of non-point source pollution load in the Three Gorges Reservoir Region, Sci. Total Environ., 493, 138146, 2014.

Shen, Z. Y., Chen, L., and Chen, T.: Analysis of parameter uncertainty in hydrological and sediment modeling using GLUE method: a case study of SWAT model applied to Three Gorges Reservoir Region, China, Hydrol. Earth Syst. Sci., 16, 121-132, https://doi.org/10.5194/hess-16-121-2012, 2012.

Shen, Z. Y., Chen, L., and Chen, T.: Effect of Rainfall Measurement Errors on Nonpoint-Source Pollution Model Uncertainty, J. Environ. Inform., 16, 14-26, 2015.

Sun, F., Chen, J., Tong, Q., and Zeng, S.: Managing the performance risk of conventional waterworks in compliance with the natural organic matter regulation, Water Res., 42, 229-237, 2008.

Vilaysane, B., Takara, K., Luo, P., Akkharath, I., and Duan, W.: Hydrological Stream Flow Modelling for Calibration and Uncertainty Analysis Using SWAT Model in the Xedone River Basin, Lao PDR, Proced. Environ. Sci., 28, 380-390, 2015.
Vrugt, J. A. and Ter Braak, C. J. F.: $\operatorname{DREAM}_{(\mathrm{D})}$ : an adaptive Markov Chain Monte Carlo simulation algorithm to solve discrete, noncontinuous, and combinatorial posterior parameter estimation problems, Hydrol. Earth Syst. Sci., 15, 3701-3713, https://doi.org/10.5194/hess-15-3701-2011, 2011.

Vrugt, J. A., Gupta, H. V., Bouten, W., and Sorooshian, S.: A Shuffled Complex Evolution Metropolis algorithm for optimization and uncertainty assessment of hydrologic model parameters, Water Resour. Res., 39, 113-117, 2003.

Westerberg, I. K., Guerrero, J.-L., Younger, P. M., Beven, K. J., Seibert, J., Halldin, S., Freer, J. E., and Xu, C.-Y.: Calibration of hydrological models using flow-duration curves, Hydrol. Earth Syst. Sci., 15, 2205-2227, https://doi.org/10.5194/hess-15-22052011, 2011.

Wu, Q., Liu, S., Cai, Y., Li, X., and Jiang, Y.: Improvement of hydrological model calibration by selecting multiple parameter ranges, Hydrol. Earth Syst. Sci., 21, 393-407, https://doi.org/10.5194/hess-21-393-2017, 2017.

Yang, J., Reichert, P., Abbaspour, K. C., Xia, J., and Yang, H.: Comparing uncertainty analysis techniques for a SWAT application to the Chaohe Basin in China, J. Hydrol., 358, 1-23, 2008.

Zhang, J., Li, Y., Huang, G., Chen, X., and Bao, A.: Assessment of parameter uncertainty in hydrological model using a MarkovChain-Monte-Carlo-based multilevel-factorial-analysis method, J. Hydrol., 538, 471-486, 2016.

Zhang, X., Liang, F., Srinivasan, R., and Van Liew, M.: Estimating uncertainty of streamflow simulation using Bayesian neural networks, Water Resour. Res., 45, 257-260, 2009a.

Zhang, X., Srinivasan, R., and Bosch, D.: Calibration and uncertainty analysis of the SWAT model using Genetic Algorithms and Bayesian Model Averaging, J. Hydrol., 374, 307-317, 2009 b. 